\title{
Nanomaterials for reversion of multidrug resistance in cancer: a new hope for an old idea?
}

\author{
João Conde ${ }^{1,2 *}$, Jesús M. de la Fuente ${ }^{1}$ and Pedro V. Baptista ${ }^{2}$ \\ 1 Instituto de Nanociencia de Aragon, Universidad de Zaragoza, Zaragoza, Spain \\ 2 Departamento de Ciências da Vida, Faculdade de Ciências e Tecnologia, CIGMH, Universidade Nova de Lisboa, Campus de Caparica, Caparica, Portugal \\ *Correspondence: conde.bio@gmail.com; j.conde@campus.fct.unl.pt \\ Edited by: \\ Chiara Riganti, University of Torino, Italy \\ Reviewed by: \\ Barbara Stella, University of Turin, Italy
}

Keywords: nanoparticles, nanomaterials, multidrug resistance, cancer, drug delivery, siRNAs

Cancer is a major cause of mortality in the modern world, with more than 10 million new cases every year. This outline is expected to rise in the next few decades since the majority (59\%) of people diagnosed with cancer is aged over 65. In fact, around one in three people will be diagnosed with cancer throughout their lifetime (Siegel et al., 2012; Bosetti et al., 2013).

The foundation of cancer treatment is surgery, chemotherapy, radiation, antibody-blocking therapy, or a combination of these therapies (Hanahan and Weinberg, 2000). Still, many clinical chemotherapeutic and radiotherapeutic regimes are not exceptionally effective, due to multidrug resistance mechanisms, depending on the patient and the type of tumor. Therefore, there is an urgent need for more effective and valuable cancer therapeutics, in order to reduce the impact of the chemotherapeutic agents on the healthy tissues by creating more selective systems toward the cancerous cells (Alison, 2001; Perez-Tomas, 2006).

Multi-drug resistance (MDR) in cancer refers to the capacity of cancer cells to survive or become resistant from treatment of a wide variety of drugs. Cancer chemotherapy has become progressively sophisticated within the last years; however there are not any cancer therapies $100 \%$ effective against disseminated cancer. This is in fact a major problem once approximately $70 \%$ of patients do not respond to initial chemotherapy and the five-year survival rate for these patients is a low $10-30 \%$. Relapse is also frequent (Diseases, 2000).

Mechanisms of MDR include decreased uptake of drugs, reduced intracellular drug concentration by activation of the efflux transporters, modifications in cellular pathways by altering cell cycle checkpoints, increased metabolism of drugs, induced emergency response genes to impair apoptotic pathways and altered DNA repair mechanisms (Gottesman, 2002). P-glycoprotein (P-gp) is the best known membrane transporter used in MDR and has been first described in the late 1970s (Juliano and Ling, 1976). Since then, the phenomenon of cancer drug resistance became a hotspot of cancer research (Gottesman, 2002; Ullah, 2008).

Despite of the discovery of multiple new gene/protein expression signatures or factors associated with drug resistance by high throughput "-omics" technologies, none of these findings has been useful in producing efficient and specific diagnostic assays or for improvement of updated chemosensitizers. Clinical success has also been limited due to issues regarding safety, once one of the most common strategies against MDR is the development of ATP-binding cassette (ABC) transporter inhibitors, which are poorly effective and specific, increasing the toxicity associated with chemotherapy (Lage, 2008).

Nanotechnology and nanomaterials in particular, are expected to provide a range of devices to treat cancer as their sizes are well matched in size to biologic molecules and structures found inside living cells (Conde et al., 2012).

The development of nanoscale devices and structures has provided major breakthroughs in monitoring and fighting cancer (Qian et al., 2008; Ren et al., 2012; Conde et al., 2013). Cancer nanotechnology offers a wealth of safety and innovative tools to treat and diagnose cancer, such as multifunctional, targeted devices capable of bypassing crucial biological barriers and to deliver multiple therapeutic agents directly to cancer cells and adjacent tissues around tumor microenvironment (Sanvicens and Marco, 2008).

Nanoparticles (NPs) are usually produced to deliver and enhance the drug concentration inside the cancer cells, using both active and passive targeting. (NPs) are excellent tumor-targeting vehicles because of the unique inherent property of solid tumors. Numerous tumors present with defective vasculature and poor lymphatic drainage, due to their rapid growth, resulting in an enhanced permeability and retention (EPR) effect. This effect allows (NPs) to accumulate preferably at the tumor site. Once the tumor is directly connected to the main blood circulation system, multifunctional (NPs) may exploit several characteristics of the newly formed vasculature and efficiently target tumors (Conde et al., 2012; Schroeder et al., 2012). This effect constitutes one of the major advantages of (NPs) against MDR mechanisms. In fact, lipid (NPs) and nanocapsules, polymeric (NPs), metal (NPs), dendrimers and liposomes have been reported to circumvent drug resistance (Dong and Mumper, 2010) (Figure 1).

The most common nanomaterials to use against $(\mathrm{P}-\mathrm{gp})$ and $\mathrm{ABC}$ transporters resistance are non-ionic surfactants (i.e., poly(ethylene glycol), Tween $80^{\circledR}$ and Pluronics ${ }^{\circledR}$ ) that usually form hydrogel bonds with the protein to escape from the recognition and therefore increase the uptake of the nanoformulated drug (Gao et al., 2012). The ABC transporters are also expressed in normal cells and so it 


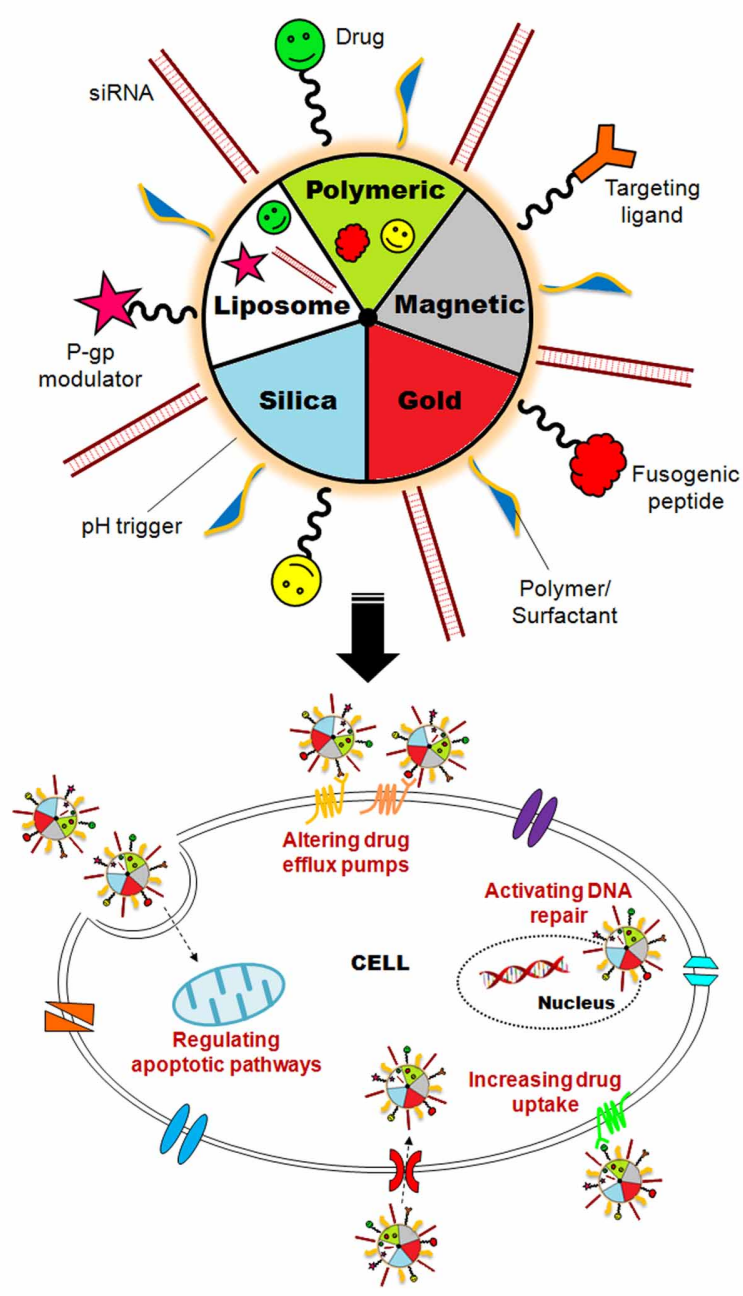

FIGURE 1 | Nanotechnology against multidrug resistance. Smart nanomaterials (i.e., liposomes, polymeric, magnetic, silica, and gold nanoparticles functionalized with MDR inhibitors and targeting moieties) can be used to circumvent essential MDR mechanisms, by increasing drug efflux and metabolism and activating DNA repair and apoptotic pathways.

is necessary to inhibit these drug efflux transporters in tumor tissues preferentially, while minimizing the inhibition in normal tissues (Patel et al., 2013).

Some of these nanomaterials have been used to overcome drug efflux by drug transporters, increasing the drug retention effect in cancer cells. These systems comprise the nanoformultation (specially with liposomes, nanodiamonds, mesoporous silica nanoparticles) of siRNAs and drugs like doxorubicin (DOX), used to hamper cancer progression, inhibiting the drug detoxification, by suppressing cell defense mechanisms, activating apoptosis and DNA repair (Minko et al., 1999; Chen et al., 2009, 2010; Chow et al., 2011).
Other drugs like vincristine, verapamil, cyclosporin, paclitaxel, oxaliplatin, cisplatin, and curcumin have been delivered using polymeric (NPs) and nanoemulsions made of surfactants (Soma et al., 2000; Devalapally et al., 2007; van Vlerken et al., 2007; Abu Lila et al., 2009; Ganta and Amiji, 2009; Song et al., 2009; Yadav et al., 2009; Aryal et al., 2010).

This combinatorial delivery of multiple drugs and MDR inhibitors opens up a huge amount of schemes to target the comprehensive mechanisms of MDR especially via the EPR effect, and at the same time it lifts up some important concerns about this approach.

Although most of these studies reported a co-delivery of multiple agents in the same nanoparticle such as siRNAs, chemotherapeutic drugs, antibodies and other MDR inhibitors, these formulations are far from achieving excellent results. This happens once the majority of drugs such as paclitaxel are hydrophobic and the MDR inhibitors like siRNAs anti-MDR associated genes are hydrophilic. This fact impairs one of the most amazing characteristic of the nanoparticle systems that is the high loading capability in the same formulation. Another problem with the loading of multiple drugs in the same nanoformulation is the molar ratios between molecules that are often incomparable and sometimes very difficult to control and quantify. This is a key element in dosage optimization and maximization of the combinatorial effects along with the nanoformulated composites (Creixell and Peppas, 2012; Gao et al., 2012).

Moreover, the interaction between chemotherapeutic drugs and MDR inhibitors (i.e., siRNAs) may occur when using coadministration. In fact, some of the drugs can strongly influence endosomal escape and siRNA recognition by the RNA Induced Silencing Complex (RISC), which are crucial obstacles/limitations for translation of RNAi into clinical setting (Lee et al., 2013). This mutual interactions between drugs and siRNAs need to be further explored and optimized.

This raises another challenge, which is the endosomal escaping of the nanoformulated drugs. Upon endocytosis the NPs-drugs usually go to the lysosomal and endosomal compartments, facing a strong acidic and enzymatic environment (Hu and Zhang, 2009). A biomolecule that bypasses the endosome would greatly increase the therapeutic effect. For example, a fusogenic peptide would promote endosomal escape by a $\mathrm{pH}$-responsive mechanism in which the peptide becomes protonated at the acidic $\mathrm{pH}$ of the endosome, destabilizing the endosomal membrane when the protonated peptide fuses with it, enabling the delivery of the siRNAs to the cytoplasm without suffering degradation. Poly(ethylene imine) (PEI) is a cationic polymer and is also used as carrier for siRNA due to its high siRNA binding capacity and its proton sponge effect for endosomal escape (Creixell and Peppas, 2012). 
However, some effective nanoparticle formulations (in particular liposomes and micelles) have been reported using the endocytic uptake of drug to avoid MDR (Thierry et al., 1993; Huwyler et al., 2002; Rapoport et al., 2002). This occurs since formulated/encapsulated drugs are more effective than free drugs, as sometimes they have more cytotoxicity to resistant cancer cells and/or improved internalization yields (Huwyler et al., 2002; Gabizon et al., 2003).

An alternative to the use of siRNA-NPs are the antisense oligodeoxynucleotides nanoformulations, once are more stable in biological matrices and have more resistance to enzymatic degradation (Conde et al., 2010; Wang et al., 2010).

In addition of active targeting, the long circulating half-life and drug release kinetics are important aspects of an effective strategy to combat MDR. In fact, the rate of drug release from the NP decides their therapeutic effectiveness. When fast drug release may lead to their lost while in blood stream circulation, a slow kinetic may predispose cancer cells to more resistance once they may not compete with the drug efflux pumps (Hu and Zhang, 2009).

To increase life-time, long circulatingNPs are usually coated with poly(ethylene glycol) (PEG), which has the ability to circulate for a prolonged period of time to allow efficient target of a particular cell/tissue/organ and retards the uptake by the macrophages and monocytes, from the reticuloendothelial system (RES) (Kommareddy et al., 2005; Uchida et al., 2005).

Concerning the stimuli-responsive drug release, $\mathrm{pH}$-sensitive triggers are the most common strategies once tumoral tissues are well known for presenting acidic conditions, as well as the lysosomal and endosomal compartments, which represent a strong advantage for selective drug release. Several nanoformulations of $\mathrm{pH}$-triggered drug release have been reported, especially those using $\mathrm{pH}$ sensitive liposomes (Fattal et al., 2004), polymeric (NPs) (Roux et al., 2002), micelles (Lee et al., 2008) and hydrogels (Griset et al., 2009). Generally, (NPs) are coated with biomolecules (i.e., phosphatidylethanolamine, poly(ethylene glycol), poly(L-histidine), poly(L-lactic acid), polymethacrylates, poly(amidoamine)) that, when protonated, destabilize the formulation (that become leaky in acidic environment) leading to the accelerated/controlled intracellular drug release. This kinetic release overwhelms the (P-gp) drug efflux pumps (Hu and Zhang, 2009).

Although the use of organic (NPs) such as liposomes, lipids, micelles, and polymeric (NPs) constitute the major strategy to deliver high amounts of drugs and MDR inhibitors, the use of inorganic (NPs) to reverse $\mathrm{MDR}$ in cancer has also been reported.

The most frequent systems usually combine silica (Rigby, 2007; Chen et al., 2009), magnetic (Cheng et al., 2011; Singh et al., 2011; Klostergaard and Seeney, 2012) or gold (NPs) (Dreaden et al., 2012; Tomuleasa et al., 2012) with specific drugs and siRNAs. The silica (NPs) are ideal candidates for the loading of large amounts of drugs and other components, due to high surface area to volume ratio and large pore volume. The magnetic (NPs) allow for physical/magnetic enhancement of the passive mechanisms for the accumulation of magnetic-responsive (NPs) into tumor tissue, leading to increase cellular uptake. Gold (NPs) also have shape/size-dependent optoelectronic properties and the endosomal-based route for gold nanoparticle cellular uptake is one of the main advantages for overcome MDR (Ayers and Nasti, 2012; Creixell and Peppas, 2012).

As described above, an extensive knowledge has been gathered in the emerging field of nanomaterials to overcome multidrug resistance mechanisms. Many of the drugs available to circumvent MDR were in the past unavailable to target this mechanism due to low solubility and/or stability. Nanomaterials made these drugs a possible strategy to target MDR. However, many anti-cancer drugs have never been used in nanomedicine (Dong and Mumper, 2010). Moreover, nanomaterials have also provided an effective platform to deliver high loads of drugs (thus lower chances of resistance) in a specific and controlled way (using $\mathrm{pH}$-responsive and stimuli-sensitive), with surface-modified to improve circulation time, preventing the uptake by the RES. Another advantage of using nanomaterials for MDR regression is the drastic reduction of the $\operatorname{IC}(50)$ value for most of the nanoformulated drugs, reducing the clinical doses of the conventional chemotherapeutic agents, which show high levels of cytotoxicity. This allows the expansion of the anticancer therapeutic window.

However, many challenges involved in biocompatibility and specificity of nanomaterials against MDR need to be further investigated. Other crucial issues embrace the fact that agents that can reduce MDR in vitro, are sometimes useless in patients, probably because some drugs get entrapped in circulation by serum proteins, for example. In addition, new nanoparticle-based targeting moieties such as antibodies, minibodies and peptides should be explored as an additive effect of EPR.

Moreover, it is important to realize that cancer cells need to lose their chemoprotective features mediated by MDR genes, at the same time the chemotherapy-sensitive non-cancerous cells (i.e., bone marrow stem cells) need to be protected from the effects of chemotherapeutic agents. In fact, the destruction of these cells constitutes the single most important dose-limiting toxicity factor in cancer therapy. If we could repopulate the blood system with chemoresistant blood cells the patients could receive higher doses of anticancer agents than could be given normally.

This raises the important issue of the specificity and toxicity of nanomaterials as one of the major obstacles to treat multidrug-resistant tumors. Therefore, any nanoformulation that target MDR must do so in a way that is tumoral specific, in order not to affect the normal function of healthy cells. This may be achieved using, for example, effective targeting ligands combined with valuable pro-drugs.

Some of the described drug nanoformulations here are now in human clinical trials. So it is therefore predictable that nanomaterials against MDR will eventually become commonplace in the oncology clinic in the near future.

\section{AUTHOR CONTRIBUTIONS}

João Conde conceptualized the manuscript and wrote the draft. Pedro V. Baptista and Jesús M. de la Fuente revised the manuscript. All authors contributed in the revision process. All authors read and approved the final manuscript. 


\section{ACKNOWLEDGMENTS}

Authors thank ERANET-NANOSCIERA NANOTRUCK project for financial support. JC acknowledges FCT grant (SFRH/BD/62957/2009). Jesús M. de la Fuente thanks ARAID and Fondo Social Europeo for financial support. Pedro V. Baptista thanks CIGMH/FCT/MCES (PEst-OE/SAU/UI0009/2011).

\section{REFERENCES}

Abu Lila, A. S., Kizuki, S., Doi, Y., Suzuki, T., Ishida, T., and Kiwada, H. (2009). Oxaliplatin encapsulated in PEG-coated cationic liposomes induces significant tumor growth suppression via a dual-targeting approach in a murine solid tumor model. J. Control. Release 137, 8-14. doi: 10.1016/j.jconrel.2009.02.023

Alison, M. R. (2001). Cancer. London: eLS John Wiley and Sons, Ltd. doi: 10.1038/npg.els.0001471

Aryal, S., Hu, C. M. J., and Zhang, L. F. (2010). Polymer-Cisplatin conjugate nanoparticles for acid-responsive drug delivery. ACS Nano. 4, 251-258. doi: 10.1021/nn9014032

Ayers, D., and Nasti, A. (2012). Utilisation of nanoparticle technology in cancer chemoresistance. J. Drug Deliv. 2012, 265691. doi: 10.1155/2012/ 265691

Bosetti, C., Bertuccio, P., Malvezzi, M., Levi, F., Chatenoud, L., Negri, E., et al. (2013). Cancer mortality in Europe, 2005-2009, and an overview of trends since 1980. Ann. Oncol. 24, 2657-2671. doi: 10.1093/annonc/mdt301

Chen, A. M., Zhang, M., Wei, D. G., Stueber, D., Taratula, O., Minko, T., et al. (2009). Codelivery of Doxorubicin and Bcl-2 siRNA by mesoporous silica nanoparticles enhances the efficacy of chemotherapy in multidrug-resistant cancer cells. Small 5, 2673-2677. doi: 10.1002/smll. 200900621

Chen, Y. C., Wu, J. Z. J., and Huang, L. (2010). Nanoparticles targeted with NGR motif deliver c-myc siRNA and doxorubicin for anticancer therapy. Mol. Ther. 18, 828-834. doi: 10.1038/mt.2009.291

Cheng, J., Wang, J., Chen, B. A., Xia, G. H., Cai, X. H., Liu, R., et al. (2011). A promising strategy for overcoming MDR in tumor by magnetic iron oxide nanoparticles co-loaded with daunorubicin and 5bromotetrandrin. Int. J. Nanomed. 6, 2123-2131. doi: 10.2147/IJN.S24309

Chow, E. K., Zhang, X. Q., Chen, M., Lam, R., Robinson, E., Huang, H. J., et al. (2011). Nanodiamond therapeutic delivery agents mediate enhanced chemoresistant tumor treatment. Sci. Trans. Med. 3, 73ra21. doi: 10.1126/scitranslmed.3001713

Conde, J., de la Fuente, J. M., and Baptista, P. V. (2010). In vitro transcription and translation inhibition via DNA functionalized gold nanoparticles. Nanotechnology 21, 505101. doi: 10.1088/0957$4484 / 21 / 50 / 505101$

Conde, J., Doria, G., and Baptista, P. (2012). Noble metal nanoparticles applications in cancer. J. Drug. Deliv. 2012, 751075. doi: 10.1155/2012/751075

Conde, J., Tian, F., Hernandez, Y., Bao, C., Cui, D., Janssen, K. P., et al. (2013). In vivo tumor targeting via nanoparticle-mediated therapeutic siRNA coupled to inflammatory response in lung cancer mouse models. Biomaterials 34, 7744-7753. doi: 10.1016/j.biomaterials.2013. 06.041

Creixell, M., and Peppas, N. A. (2012). Co-delivery of siRNA and therapeutic agents using nanocarriers to overcome cancer resistance. Nano Today 7 , 367-379. doi: 10.1016/j.nantod.2012.06.013

Devalapally, H., Duan, Z. F., Seiden, M. V., and Amiji, M. M. (2007). Paclitaxel and ceramide co-administration in biodegradable polymeric nanoparticulate delivery system to overcome drug resistance in ovarian cancer. Int. J. Cancer 121 , 1830-1838. doi: 10.1002/ijc. 22886

Diseases. (2000). Cancer multidrug resistance. Nat. Biotechnol. 18(Suppl), IT18-IT20. Available online at: http://www.nature.com/nbt/ journal/v18/n10s/full/nbt1000_IT18.html

Dong, X. W., and Mumper, R. J. (2010). Nanomedicinal strategies to treat multidrugresistant tumors: current progress. Nanomedicine 5, 597-615. doi: 10.2217/nnm.10.35

Dreaden, E. C., Gryder, B. E., Austin, L. A., Defo, B. A. T., Hayden, S. C., Pi, M., et al. (2012) Antiandrogen gold nanoparticles dual-target and overcome treatment resistance in hormoneinsensitive prostate cancer cells. Bioconjug. Chem. 23, 1507-1512. doi: 10.1021/bc300158k

Fattal, E., Couvreur, P., and Dubernet, C. (2004). "Smart" delivery of antisense oligonucleotides by anionic pH-sensitive liposomes. Adv. Drug Deliv. Rev. 56, 931-946. doi: 10.1016/j.addr.2003.10.037

Gabizon, A., Shmeeda, H., and Barenholz, Y. (2003). Pharmacokinetics of pegylated liposomal doxorubicin-Review of animal and human studies. Clin. Pharmacokinet. 42, 419-436. doi: 10.2165/00003088-200342050-00002

Ganta, S., and Amiji, M. (2009). Coadministration of paclitaxel and curcumin in nanoemulsion formulations to overcome multidrug resistance in tumor cells. Mol. Pharm. 6, 928-939. doi: $10.1021 / \mathrm{mp} 800240 \mathrm{j}$

Gao, J., Feng, S. S., and Guo, Y. J. (2012) Nanomedicine against multidrug resistance in cancer treatment. Nanomedicine 7, 465-468. doi: $10.2217 / \mathrm{nnm} .12 .11$

Gottesman, M. M. (2002). Mechanisms of cancer drug resistance. Annu. Rev. Med. 53, 615-627. doi: 10.1146/annurev.med.53.082901.103929

Griset, A. P., Walpole, J., Liu, R., Gaffey, A., Colson, Y. L., and Grinstaff, M. W. (2009). Expansile nanoparticles: synthesis, characterization, and in vivo efficacy of an acid-responsive polymeric drug delivery system. J. Am. Chem. Soc. 131, 2469-2471. doi: 10.1021/ja807416t

Hanahan, D., and Weinberg, R. A. (2000). The hallmarks of cancer. Cell 100, 57-70. doi: 10.1016/S0092-8674(00)81683-9

Hu, C. M. J., and Zhang, L. F. (2009). Therapeutic nanoparticles to combat cancer drug resistance. Curr. Drug Metab. 10, 836-841. doi: 10.2174/138920009790274540

Huwyler, J., Cerletti, A., Fricker, G., Eberle, A. N., and Drewe, J. (2002). By-passing of P-glycoprotein using immunoliposomes. J. Drug Target. 10, 73-79. doi: 10.1080/10611860290007559

Juliano, R. L., and Ling, V. (1976). A surface glycoprotein modulating drug permeability in Chinese hamster ovary cell mutants. Biochim. Biophys. Acta 455, 152-162. doi: 10.1016/0005-2736 (76) $90160-7$

Klostergaard, J., and Seeney, C. E. (2012). Magnetic nanovectors for drug delivery. Nanomed. Nanotechnol. Biol. Med. 8, S37-S50. doi: 10.1016/j. nano.2012.05.010

Kommareddy, S., Tiwari, S. B., and Amiji, M. M. (2005). Long-circulating polymeric nanovectors for tumor-selective gene delivery. Technol. Cancer Res. Treat. 4, 615-625.

Lage, H. (2008). An overview of cancer multidrug resistance: a still unsolved problem. Cell. Mol. Life Sci. 65, 3145-3167. doi: 10.1007/s00018 008-8111-5

Lee, E. S., Gao, Z. G., and Bae, Y. H. (2008). Recent progress in tumor $\mathrm{pH}$ targeting nanotechnology. J. Control. Release 132, 164-170. doi: 10.1016/j.jconrel.2008.05.003

Lee, J. M., Yoon, T. J., and Cho, Y. S. (2013). Recent developments in nanoparticle-based siRNA delivery for cancer therapy. Biomed. Res. Int. 2013, 782041. doi: 10.1155/2013/782041

Minko, T., Kopeckova, P., and Kopecek, J. (1999). Comparison of the anticancer effect of free and HPMA copolymer-bound adriamycin in human ovarian carcinoma cells. Pharm. Res. 16, 986-996. doi: 10.1023/A:1018959029186

Patel, N. R., Pattni, B. S., Abouzeid, A. H., and Torchilin, V. P. (2013). Nanopreparations to overcome multidrug resistance in cancer. Adv. Drug Deliv. Rev. doi: 10.1016/j.addr.2013.08.004. [Epub ahead of print]

Perez-Tomas, R. (2006). Multidrug resistance: retrospect and prospects in anti-cancer drug treatment. Curr. Med. Chem. 13, 1859-1876. doi: 10.2174/092986706777585077

Qian, X. M., Peng, X. H., Ansari, D. O., Yin-Goen, Q., Chen, G. Z., Shin, D. M., et al. (2008). In vivo tumor targeting and spectroscopic detection with surface-enhanced raman nanoparticle tags. Nat Biotechnol. 26, 83-90. doi: 10.1038/nbt1377

Rapoport, N., Marin, A., Luo, Y., Prestwich, G. D., and Muniruzzaman, M. (2002). Intracellular uptake and trafficking of pluronic micelles in drugsensitive and MDR cells: effect on the intracellular drug localization. J. Pharm. Sci. 91, 157-170. doi: 10.1002/jps.10006

Ren, Y., Cheung, H. W., von Maltzhan, G., Agrawal, A. Cowley, G. S., Weir, B. A., et al. (2012). Targeted Tumor-Penetrating siRNA Nanocomplexes for Credentialing the Ovarian Cancer Oncogene ID4 Sci. Trans. Med. 4, 147ral12. doi: 10.1126/scitranslmed.3003778

Rigby, P. (2007). Silica nanoparticles target cancer cells. Nano Today 2, 12-12. doi: 10.1016/S17480132(07)70110-X

Roux, E., Francis, M., Winnik, F. M., and Leroux J. C. (2002). Polymer based pH-sensitive carriers as a means to improve the cytoplasmic delivery of drugs. Int. J. Pharm. 242, 25-36. doi: 10.1016/S0378-5173(02)00183-7

Sanvicens, N., and Marco, M. P. (2008). Multifunctional nanoparticles-properties and prospects for their use in human medicine. Trends Biotechnol. 26, 425-433. doi: 10.1016/j. tibtech.2008.04.005

Schroeder, A., Heller, D. A., Winslow, M. M., Dahlman, J. E., Pratt, G. W., Langer, R., et al. 
(2012). Treating metastatic cancer with nanotechnology. Nat. Rev. Cancer 12, 39-50. doi: $10.1038 / \operatorname{nrc} 3180$

Siegel, R., Naishadham, D., and Jemal, A. (2012) Cancer statistics, 2012. CA. Cancer J. Clin. 62, 10-29. doi: 10.3322/caac. 20138

Singh, A., Dilnawaz, F., and Sahoo, S. K. (2011). Long circulating lectin conjugated paclitaxel loaded magnetic nanoparticles: a new theranostic avenue for leukemia therapy. PLoS ONE 6:e26803. doi: 10.1371/journal.pone.0026803

Soma, C. E., Dubernet, C., Bentolila, D., Benita, S., and Couvreur, P. (2000). Reversion of multidrug resistance by co-encapsulation of doxorubicin and cyclosporin A in polyalkylcyanoacrylate nanoparticles. Biomaterials 21, 1-7. doi: 10.1016/S01429612(99)00125-8

Song, X. R., Cai, Z., Zheng, Y., He, G., Cui, F. Y., Gong, D. Q., et al. (2009). Reversion of multidrug resistance by co-encapsulation of vincristine and verapamil in PLGA nanoparticles. Eur. J. Pharm. Sci. 37, 300-305. doi: 10.1016/j.ejps.2009. 02.018

Thierry, A. R., Vige, D., Coughlin, S. S., Belli, J. A., Dritschilo, A., and Rahman, A. (1993). Modulation of doxorubicin resistance in multidrug-resistant cells by liposomes. FASEB J. 7, 572-579.

Tomuleasa, C., Soritau, O., Orza, A., Dudea, M., Petrushev, B., Mosteanu, O., et al.
(2012). Gold nanoparticles conjugated with cisplatin/doxorubicin/capecitabine lower the chemoresistance of hepatocellular carcinomaderived cancer cells. J. Gastrointestin. Liver Dis. 21, 187-196. Available online at: http://www.jgld.ro/2012/2/15.html

Uchida, K., Otsuka, H., Kaneko, M., Kataoka, K. and Nagasaki, Y. (2005). A reactive poly(ethylene glycol) layer to achieve specific surface plasmon resonance sensing with a high $\mathrm{S} / \mathrm{N}$ ratio: the substantial role of a short underbrushed PEG layer in minimizing nonspecific adsorption. Anal. Chem. 77, 1075-1080. doi: 10.1021/ ac0486140

Ullah, M. F. (2008). Cancer Multidrug Resistance (MDR): a major impediment to effective chemotherapy. Asian Pacific J. Cancer Prevent. 9, 1-6. Available online at: http://www.apocpcontrol. org/page/apjcp_issues_view.php?sid=Entrez:Pub Med\&id=pmid:18439063\&key=2008.9.1.1

van Vlerken, L. E., Duan, Z. F., Seiden, M. V., and Amiji, M. M. (2007). Modulation of intracellular ceramide using polymeric nanoparticles to overcome multidrug resistance in cancer. Cancer Res. 67, 4843-4850. doi: 10.1158/0008-5472.CAN-061648

Wang, J. Q., Tao, X. Y., Zhang, Y. F., Wei, D. Z., and Ren, Y. H. (2010). Reversion of multidrug resistance by tumor targeted delivery of antisense oligodeoxynucleotides in hydroxypropyl-chitosan nanoparticles. Biomaterials 31, 4426-4433. doi: 10.1016/j.biomaterials.2010.02.007

Yadav, S., van Vlerken, L. E., Little, S. R., and Amiji, M. M. (2009). Evaluations of combination MDR-1 gene silencing and paclitaxel administration in biodegradable polymeric nanoparticle formulations to overcome multidrug resistance in cancer cells. Cancer Chemother. Pharmacol. 63, 711-722. doi: 10.1007/s00280-008-0790-y

Received: 22 September 2013; accepted: 08 October 2013; published online: 25 October 2013.

Citation: Conde J, De La Fuente JM and Baptista PV (2013) Nanomaterials for reversion of multidrug resistance in cancer: a new hope for an old idea? Front. Pharmacol. 4:134. doi: 10.3389/fphar.2013.00134

This article was submitted to Pharmacology of AntiCancer Drugs, a section of the journal Frontiers in Pharmacology.

Copyright (c) 2013 Conde, De La Fuente and Baptista. This is an open-access article distributed under the terms of the Creative Commons Attribution License (CC BY). The use, distribution or reproduction in other forums is permitted, provided the original author(s) or licensor are credited and that the original publication in this journal is cited, in accordance with accepted academic practice. No use, distribution or reproduction is permitted which does not comply with these terms. 